\title{
Camouflaging nursing research-related tasks in clinical practice-Experiences of newly-graduated masters of science in nursing
}

\author{
Connie Bøttcher Berthelsen, Marianne Vamosi, Bente Martinsen \\ Research Unit of Nursing and Healthcare, Institute of Health, Nursing, Aarhus University, Denmark
}

Received: November 7, 2019

DOI: $10.5430 /$ jnep.v10n3p42
Accepted: November 18, 2019 Online Published: November 25, 2019

URL: https://doi.org/10.5430/jnep.v10n3p42

\begin{abstract}
Objective: To explore and describe how newly-graduated Masters of Science in Nursing experienced engaging in nursing research-related tasks in daily clinical practice.

Methods: Fifteen nurses withholding a Masters of Science in Nursing degree were recruited from our longitudinal cohort study and interviewed six months after graduation in December $2016(\mathrm{n}=10)$ and in December $2017(\mathrm{n}=5)$, respectively. Data were analysed using Graneheim and Lundmann's qualitative manifest and latent content analysis. Lincoln and Guba's four criteria of trustworthiness were followed.

Results: The main theme of the overall interpretation was Camouflaging nursing research-related tasks in clinical practice. The main theme describe the Master of Science in Nursing graduates as highly motivated to use their new academic skills in clinical practice and how they have to hide their engagement in research due to the barriers, which are outlined in the three themes: the position as time restrainer, the management as gatekeeper, and the nursing culture as norm setter.

Conclusions: The study contributes with knowledge on how the Master of Science in Nursing graduates struggle to use their academic skills in clinical practice and how they felt the need to camouflage their commitment in research because it was not well reputed among their colleagues.
\end{abstract}

Key Words: Clinical practice, Master of science in nursing, Nursing culture, Qualitative content analysis, Research utilisation

\section{INTRODUCTION}

The focus on implementing evidence-based knowledge into clinical nursing practice has increased during recent decades, as there has been an aim to further improve patient care and strengthen the nursing profession. However, studies show a lack of research utilisation among nurses in clinical practice. This is primarily caused by several barriers experienced by the nurses, such as lack of time, lack of authority to change practice and lack of multiple implementation facilities. ${ }^{[1,2]}$
One of the most well-known and investigated barriers against research utilisation is nurses' lack of research knowledge and ability. ${ }^{[1,3-5]}$ Clearly this could be caused by nurses' lack of education in research utilisation and implementation of evidence-based knowledge. Most studies exploring and describing nurses' barriers to research utilisation are based on data from registered nurses, who did not learn much about research at nursing school. ${ }^{[6,7]}$

The educational and course-related content of the Master

\footnotetext{
*Correspondence: Connie Bøttcher Berthelsen; Email: cb@ph.au.dk; Address: Research Unit of Nursing and Healthcare, Institute of Health, Nursing, Aarhus University, Campus Emdrup, Tuborgvej 164, 2400 Copenhagen, Denmark.
} 
of Science in Nursing (MSN) programme varies between countries and universities in terms of the focus on academic scholarship through research methodologies and theoretical frameworks, advanced clinical care and leadership skills. In Denmark, the focus revolves around building up the MSN students' theoretical and methodological skills in nursing at Aarhus University (2019) and on integrating a strong clinical profile with practical knowledge, new skills and competencies at the University of Southern Denmark (2019).

The Master of Science in Nursing education at King's College London, UK (2019) develops students' critical thinking and knowledge of evidence-based practice, with the aim of carrying out research in a clinical setting. In the Master of Science in Nursing education at Yale University, Connecticut, USA (2019), the framework is constructed so as to prepare students to assume roles as clinicians, scholars and leaders to improve health care in clinical practice.

The MSN graduates' extended knowledge on academic scholarship and advanced clinical practice can have a positive impact on patient care. ${ }^{\left[{ }^{8-12]}\right.}$ An observational study of 422,730 patients from 300 hospitals in nine European countries showed how a $10 \%$ increase in nurses with a postgraduate degree was associated with a decrease in inpatient death within 30 days of admission by $7 \% .^{[11]} \mathrm{Ge}$ and colleagues ${ }^{[10]}$ performed a systematic review of quantitative studies to explore the impact of master's-graduate nurses on inpatient care in different medical specialties. Nine papers were included and showed how patient care was improved in palliative care, continuity of care, mental health, transition care, post-transplant care and central venous catheter care when performed by master's-graduate nurses. ${ }^{[10]}$ Another systematic review concerning the evidence that master's graduate nurses affects patient care was performed by Cotterill-Walker. ${ }^{[9]}$ The results showed five areas relating to master's-level nursing education that may ultimately affect patient care: increased confidence and self-esteem; enhanced communication; personal and professional growth; knowledge and application of theory to practice; and analytical thinking and decision-making. ${ }^{[9]} \mathrm{A}$ paper by Clark and colleagues ${ }^{[8]}$ on the value of master's graduates in the nursing workforce discussed improved patient outcomes, critical thinking and decision-making skills, and enhanced leadership qualities, which empower postgraduate nurses to challenge poor practice. In a cross-sectional survey by Drennan, ${ }^{[12]}$ enhanced leadership skills and management capabilities were also found to be prominent among nurses who had a master's degree.

The comprehensive knowledge on academic scholarship through research methodologies and theoretical frameworks have also shown evidence for critical thinking as well as

Published by Sciedu Press research utilisation and implementation abilities. In a questionnaire study of factors influencing the development of evidence-based nursing practice for 68 Canadian registered nurses and nurse practitioners, Baird and Miller ${ }^{[13]}$ found significant differences between the meaning of educational level: expert nurses with a higher level of education reported being more skillful at synthesising and applying information from research findings to their nursing practice. However, Oostveen and colleagues ${ }^{[14]}$ found perceived barriers among academic nurses who combined clinical practice and academic work, such as a lack of opportunities for nurses to have academic careers, an underuse of nurse academics and the absence of a structure for nurses working with academics in clinical practice.

Knowledge is sparse concerning MSN graduates' experiences in stepping into clinical practice with extended knowledge on research methodology and methods from their academic education. In order to fully understand the use of competencies we wanted to explore how they experienced the opportunities to engage in and utilise nursing researchrelated tasks in clinical practice and how they perceive the challenges in clinical nursing practice. The aim of this study was therefore to explore and describe how newly-graduated Masters of Science in Nursing experienced engaging in and utilising nursing research-related tasks in daily clinical practice.

\section{Methods}

\subsection{Design}

A qualitative study was performed based on Graneheim and Lundman' ${ }^{[15]}$ manifest and latent content analysis of semistructured interviews with the MSN graduates. The design was chosen to provide a description as well as an interpretation of the interviews in order to describe the MSN graduates' various realities. ${ }^{[15]}$

\subsection{Participants}

The participants in this study were recruited from our longitudinal cohort study ${ }^{[16]}$ aiming to describe the development of MSN graduates' utilization and improvement of nursing research-related tasks and knowledge in daily clinical practice. Using purposeful sampling during our data collection in the cohort study, the MSN graduates were invited to participate in the interviews approximately six months after graduation. Thirty-eight MSN graduates were invited to participate in the interviews and 15 of these accepted the invitation.

The 15 participating MSN graduates were women aged 26 to 44. They had graduated as Bachelors in Nursing three to 19 years prior to the study, and five of them had attained 
additional qualifications after nursing school, such as clinical specialist educations and a bachelor in nature science. Eight MSN graduates were employed in full-time research and development positions, five MSN graduates were employed in dual part-time positions between research and patient care, and two held full-time positions in patient care (see Table 1).

\subsection{Data collection}

Data were collected through 15 semi-structured qualitative interviews based on an interview guide. The questions were based on the cohort questionnaire ${ }^{[16]}$ to provide a qualitative picture of the participants' perceptions of their opportunities to engage in nursing research-related tasks in daily clinical practice. The questions revolved around the participants' experiences of their daily work in clinical practice subsequent to graduating as Masters of Science in Nursing, their colleagues and management's support and interest in nursing research, and their engagement in nursing research-related tasks in daily clinical practice.

The interviews were conducted in December $2016(n=10)$ and in December $2017(n=5)$ by the first and last author in the MSN graduates' hospital departments or over Skype. The 15 interviews were transcribed verbatim by the first and last author and by a student assistant.

Table 1. A condensed description of the 15 participating MSN graduates

\begin{tabular}{lllll}
\hline Graduates & $\begin{array}{l}\text { Years of } \\
\text { age }\end{array}$ & $\begin{array}{l}\text { Years as educated } \\
\text { nurse }\end{array}$ & $\begin{array}{l}\text { Additional education after } \\
\text { bachelor in nursing }\end{array}$ & $\begin{array}{l}\text { Division of positions in percentage } \\
\text { (Research time/patient care) }\end{array}$ \\
\hline A & 26 & 3 & None & $0 / 100$ \\
B & 27 & 4 & None & $60 / 40$ \\
C & 31 & $51 / 2$ & None & $100 / 0$ \\
D & 38 & 13 & Specialist in gynecology nursing & $50 / 50$ \\
E & 36 & 8 & None & $100 / 0$ \\
F & 29 & 6 & None & $0 / 100$ \\
G & 38 & 14 & None & $100 / 0$ \\
H & 44 & 19 & Specialist in intensive care & $50 / 50$ \\
I & 28 & 7 & None & $50 / 50$ \\
J & 33 & 10 & None & $100 / 0$ \\
K & 35 & 10 & Specialist in critical care & $100 / 0$ \\
L & 31 & 7 & None & $100 / 0$ \\
M & 41 & 3 & Bachelor in science of nature & $60 / 40$ \\
N & 36 & 12 & Specialist in clinical teaching & $100 / 0$ \\
O & 38 & 14 & None & $100 / 0$ \\
\hline
\end{tabular}

\subsection{Rigour}

Our procedure to ensure trustworthiness in this study followed Lincoln and Guba's ${ }^{[17]}$ four criteria. The first and last author performed the analysis of the data, to assure credibility, and stayed as close to the data as possible to ensure an objective interpretation. To ensure dependability, the first and last author conducted interviews, using the same interview guide, until saturation of data was reached. To assure confirmability, quotes were used to reflect the participants' voices. Quotes were chosen from a qualitative perspective according to the content and importance of the quotes (and not from a quantitative perspective) to ensure that all MSN graduates were mentioned. Quotes from all MSN graduates will therefore not (necessarily) be displayed. However, all interviews were analysed and formed part of the overall results of the final manifest and latent interpretation and analysis. In order to assure transferability, a thorough method description was provided, so other researchers would be able to evaluate the applicability of the data to other contexts.

\subsection{Data analysis}

Manifest and latent content analysis based on the classic approach of Graneheim and Lundman ${ }^{[15]}$ was chosen to provide a descriptive and explorative interpretation of the 15 interviews that constituted our unit of analysis. Both forms of analysis contain levels of interpretation but through various depths. ${ }^{[15]}$

The manifest content analysis was initiated by reading the transcribed interviews as a whole to gain insight into the visible and obvious components of the MSN graduates' descriptions. Meaning units were then identified in the interviews, consisting of pieces of text that related to each other in 
content or context, which were then condensed into smaller units of text while preserving the core meaning. The condensed units were grouped by codes, reaching a higher level of abstraction in creating the sub-categories and categories in three content areas, and the main category of "MSN graduates engagement in nursing research related tasks" (see Figure 1).

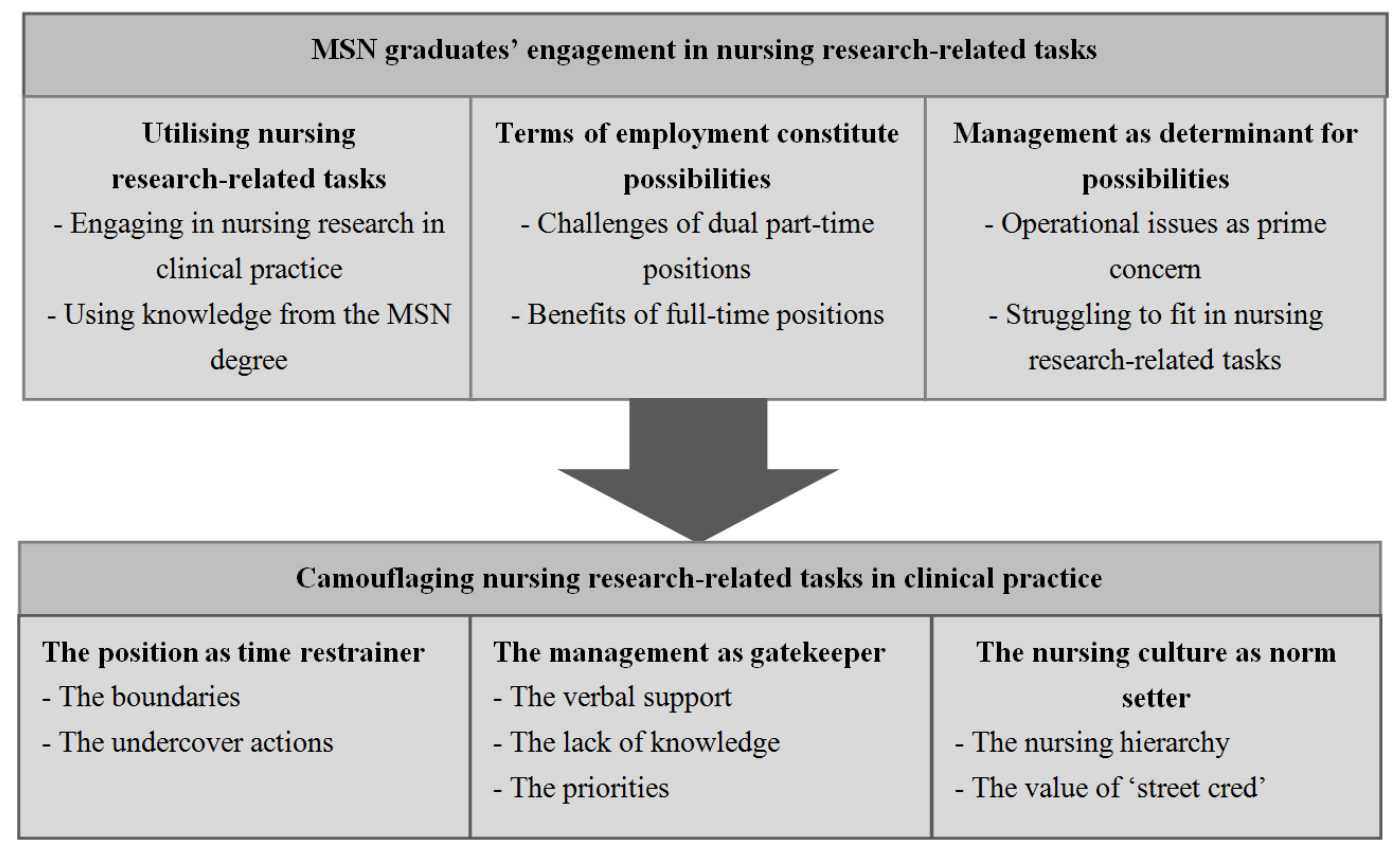

Figure 1. The overall manifest and latent interpretation and analysis

The latent analysis was followed by interpreting the underlying meaning of the interview text. ${ }^{[15]}$ The interviews were read again in the light of the manifest categories and subcategories and the underlying meanings were linked into three sub-themes and the main theme of "Camouflaging nursing research-related tasks in clinical practice" (see Figure $1)$.

The themes and sub-themes were created through manifest and latent interpretation and serve as the overall interpretation of the participants' experiences of engaging in nursing research-related tasks in daily clinical practice.

\subsection{Ethical considerations}

All participating MSN graduates received oral and written information from the interviewer about the study purpose before the interview was conducted. Their right to anonymity was explained to them, along with their right to decline the use of their interviews, and the interviewer also explained that she would maintain confidentiality regarding what was said in the interviews. All the participants signed a consent form containing their ethical and legal rights. During the presentation of the participants, we excluded job title and department type in order to ensure full anonymity for the participants. The study was approved by the Danish Data
Protection Agency (J. nr. 62908-310) and was carried out in accordance with the Code of Ethics of the World Medical Association (Declaration of Helsinki) for experiments involving humans.

\section{Findings}

The main theme of the overall interpretation of the manifest and latent content analysis of the 15 interviews was Camouflaging nursing research-related tasks in clinical practice. The theme represents the MSN graduates as highly motivated to use their new academic skills in clinical practice and shows how they anticipated meeting several barriers described in three themes covering the main theme: the position as time restrainer, the management as gatekeeper and the nursing culture as norm setter.

\subsection{The position as time restrainer \\ 3.1.1 The boundaries}

The content of the position in which the MSN graduates were employed was often a boundary itself, if the position was divided into time for research and time for patient care. In the dual part-time positions, the MSN graduates' time for nursing research-related tasks was often withdrawn if they were needed in patient care owing to the absence of their colleagues. Sometimes the MSN graduates were left with 
four hours per week to initiate research projects.

"It goes hand in hand - I mean being in close proximity to practice and development - and I don't disagree with that at all. But you can't plan anything (...) and they have to be aware that dragging me into patient care has its consequences" (Graduate D, 13 years as educated nurse with a specialist education in gynecology nursing)

The limited time for research meant that the MSN graduates were frustrated and constantly felt uncertain about which tasks to prioritise. Repeatedly the research-related work was overridden in favour of other operational tasks. The MSN graduates' motivation was driven by their eagerness to learn more about research, to study the implementation forms and to get rid of routines based on outdated knowledge. However, the MSN graduates were aware of the limited time and space for research and the fact that they needed to start somewhere.

"We have large units but there are very few research projects in nursing. And I think it's a big limitation. But you have to start somewhere, you know" (Graduate C, 5.5 years as educated nurse with no additional education beside the MSN)

The MSN graduates described an inner passion for nursing research-related tasks and were determined to explore the boundaries of the department to find out how far they could go to get permission to work on research. The candidates explained how they needed knowledgeable colleagues to guide their way in the research-related tasks but there were seldom any available. The MSN graduates explained how collaboration in research and support from peers was important for how they managed and conducted the nursing research-related tasks.

\subsubsection{The undercover actions}

Because of their limited time for research and in order to stay motivated, the MSN graduates conducted the nursing research-related tasks in their spare time or while on their shifts. The MSN graduates felt a necessity for "sneaking" around with their research in order to get it done. They often used their spare time to write articles, prepare for nursing symposiums and develop projects for clinical practice.

"My unit nurse was determined that I should work on a project about patients' relatives. I think I got just about one day for that. And it's great that they give you some time. Otherwise, I've had to do most of it at home" (Graduate A, three years as educated nurse with no additional education beside the MSN)

The MSN graduates recognised that working at home was the way to work in clinical practice but felt distressed about the strain on their families and time away from them. The MSN graduates in dual part-time positions sometimes worked shifts to take care of patients and often used their breaks to work on development projects initiated by the departments. The undercover actions of research were often a burden for the MSN graduates and some of them stated they were willing to work for the wage of a registered nurse, just to have a chance to do research.

\subsection{The management as gatekeeper}

\subsubsection{The verbal support}

The MSN graduates experienced the nursing management as verbally supportive of their engagement in nursing researchrelated tasks through encouragement and goodwill. However, additional resources, such as time and money, rarely accompanied the support. The MSN graduates' explained how their was only a very limited budget for research in clinical practice, which had consequences for their participation in nursing research-related tasks.

"There is no development! There is no sparring for the newlyeducated nurses and we don't get any teaching (...) I was told that there just isn't any money for it" (Graduate F, six years as educated nurse with no additional education beside the MSN)

The MSN graduates explained how their ideas of developing and implementing research in clinical practice often were met with high enthusiasm by their head nurses but no financial support.

"They're all in favour of us. There are just a few things that need sorting out, like the financial side of things. Um... they don't have any money for it at the moment" (Graduate $\mathrm{M}$, three years as educated nurse with a Bachelor degree in science of nature)

The MSN graduates remained frustrated by their inability to use the knowledge they had attained during their Master of Science in Nursing degree. However, they felt satisfied knowing that their head nurse gave them moral support.

\subsubsection{The lack of knowledge}

Some MSN graduates experienced that they had been hired because of the convenience and high status of having an academic nurse in the department, rather than any genuine need for their academic skills. Often the nursing management was unaware of how to use their specific academic skills in clinical practice,

"I think the head nurse enjoys having MSN graduates in the department, and then she needs to figure out how and what she's allowed to use us for, and what she can afford to use us for" (Graduate L, seven years as educated nurse with no additional education beside the MSN) 
The nursing management often did not know which tasks to include in the MSN graduates' positions and some graduates were given the opportunity to develop the content of their positions themselves. Also the MSN graduates could feel pressured by the assignment and felt left alone.

“(... but when I came back I was given the same tasks as before and told that I should let them know if I could think of anywhere I could use my master's education. And I thought that was very odd" (Graduate E, eight years as educated nurse with no additional education beside the MSN )

Despite its lack of knowledge regarding the use of academic skills in clinical practice, the nursing management was still open to suggestions. Some MSN graduates succeeded in making concrete changes in clinical practice in collaboration with the management; for instance, mattresses for pressure ulcer prevention were implemented for patients after heart surgery.

\subsubsection{The priorities}

The MSN graduates described how low priority was often given to nursing research-related tasks by the nursing management, in comparison with operational issues in running the department. The MSN graduates - especially those who held dual part-time positions - explained how they needed time to perform the nursing research-related tasks, and often had to take care of patients when working shifts.

“(... ) you have a passion for something in the department (...) and that's what gets cancelled when the place is busy. And that challenge is even bigger when you work shifts" (Graduate D, 13 years as educated nurse with a specialist education in gynecology nursing)

The MSN graduates saw how the nursing management experienced pressure from assignments and implementation dictated by senior management at the hospital. They explained how they had seen the nursing management struggle to try to acquire resources for research in clinical nursing practice but were often disappointed. The lack of knowledge regarding the use of academic skills in clinical practice among nursing managers could make it difficult for the MSN graduates to argue for more resources for nursing research-related tasks.

\subsection{The nursing culture as norm setter}

\subsubsection{The nursing hierarchy}

The types of tasks assigned to the nurses determined their place in the hierarchy and the amount of respect they gained from their nursing colleagues in clinical practice. The MSN graduates were well aware of their place at the bottom of the hierarchy, because their work did not primarily revolve around patient care.

Published by Sciedu Press
"There are actually some rules and a hierarchy, and that's where I need to try to fit in. Which is perfectly normal (...) however, it kind of slows you down. So I think you have to be a strong person in order to make your case" (Graduate A, three years as educated nurse with no additional education beside the MSN )

The MSN graduates tried to fit into the hierarchy by being amenable regarding new assignments and ideas, and they knew that the best approach was to obtain a sense of how things were done in the specific department. The MSN graduates explained how the most experienced nurses wanted to care for the patients "in the usual way" without any changes, and they often had the last word regarding decisions. The nursing research-related tasks were often new for the department nurses and the MSN graduates found it difficult to find a position in the hierarchy of clinical practice when their tasks differed from the norm of taking care of the patients even though they had several years of experience in practice.

"The dual part-time position is great, when you're also caring for patients. It's a great help. Because as soon as you turn up with a different academic qualification you stand out as being different." (Graduate B, four years as educated nurse with no additional education beside the MSN)

Newly-graduated master's nurses with no prior attachment to the department found it difficult fitting in and establishing an appropriate place in the hierarchy. However, combining the nursing research-related tasks with patient care seemed to help.

\subsubsection{The value of 'street cred'}

'Street cred' was attained when the candidates performed patient-related tasks, were visible in clinical practice and avoided differing from the norms of the nursing hierarchy. One way of fitting into clinical practice was to wear a nursing uniform rather than 'normal' clothing. The MSN graduates perceived it as a strong feature.

"Our Clinical Nurse Specialist used to sit with the management, wearing normal clothes, and it's really the little things that matter (...). There's a big difference when I turn up in my white coat, even on my administrative days. I think it's helped a lot" (Graduate B, four years as educated nurse with no additional education beside the MSN)

Being visible in the department was also a strategy the MSN graduates used to gain a respected place in the hierarchy, instead of having an office on the management floor. The MSN graduates explained how nursing colleagues talked about not knowing who was on the management floor and what the nurses there were actually doing. 
"I actually think it's important to maintain relations with the nurses in the clinic instead of just sitting in my office on the 7 th floor, otherwise I would be out of the picture. I would be seen as someone who got tired of nursing care and chose a desk job. And that's not the case" (Graduate O, 14 years as educated nurse with no additional education beside the MSN)

The MSN graduates were aware of the value of 'street cred' and how they had to adjust to clinical practice in order to perform nursing research-related tasks and to find a position for themselves in the department. In some cases the MSN graduates offered bedside teaching to their nursing colleagues as a way to use their academic knowledge in close proximity to patient care. The MSN graduates received excellent support from their nursing colleagues, who experienced the bedside teaching as a way to learn best practice close to the patients. They experienced this set-up as a perfect way to combine nursing research-related tasks with clinical practice care.

\section{Discussion}

The findings show that the MSN graduates struggled with their professional identity, no regards to their years of being educated as nurses og having specialist educations beside the MSN. On the one hand, they strove to be part of the nursing group, and on the other hand, they had a strong desire to use their new skills. Hamran ${ }^{[18]}$ writes that nursing communities are characterised by a continuous shared responsibility for patient care. Nurses' cooperation arises from the actual nursing care and is conditioned by an inner connection between the tasks involving the patients and the organisation of the work. However, the workplace environment is not built on a traditional organisation model, but rather on the shared responsibility for 'helping' and 'taking care' of sick people. ${ }^{[18]}$ This perspective suggests that the MSN graduates may be excluded from the nursing community when their main tasks do not include direct nursing care. In a study by Oostveen and colleagues ${ }^{[14]}$ on how nurses combine clinical practice with academic work, the results showed how direct-patient care gains focus in clinical practice, while academic tasks are underappreciated. Renolen and colleagues ${ }^{[6]}$ also showed how limited capacity was used as a reason to "off-track" certain activities, such as research and implementation of evidence, in order to "on-track" patient care activities. Hamran' ${ }^{[18]}$ contribution may explain why the MSN graduates tried hard to gain 'street cred' through direct care. In nursing culture, it is common to prove one's worth through practical care and nurses like to work in groups. ${ }^{[18]}$ Traditionally it is not positive to be perceived as a 'paper pusher' when you are a nurse working in a clinical setting, ${ }^{[18]}$ which could explain why the MSN graduates' positions may involve a conflict between being part of the nursing group and being a nurse with development and research duties. This understanding of community among clinical nurses may also explain why it was experienced as more troublesome for newly-employed nurses with no prior connection to the ward to legitimise their interest in research and development tasks.

The phenomenon of MSN graduates camouflaging nursing research-related tasks in clinical practice can be discussed in terms of Honneth's theory ${ }^{[19]}$ on recognition as an important aspect of cooperative relationship. According to Honneth, ${ }^{[19]}$ recognition is differentiated into three spheres of recognition: (1) the sphere of privacy, as we know from family and friends, (2) the judicial sphere and (3) the sphere of solidarity that covers cultural, political and working communities. Recognition within these three spheres constitutes an ontogenetic step in the development of an individual, since it is necessary that the individual experience all three forms of recognition to be fully individuated. ${ }^{[19]}$ When it comes to cooperation between professional groups, recognition in the sphere of solidarity is relevant. Honneth ${ }^{[19]}$ stresses that coming to relate to oneself necessarily involves experiencing recognition from others, since one's relationship to oneself is an intersubjective process in which one's attitude towards oneself emerges in one's encounter with another's attitude towards oneself. Nurses conducting research-related tasks in clinical practice often experience a lack of recognition from their colleagues, who downplay the importance of time for research. ${ }^{[6,14,20]}$ In our study, the master's MSN graduates were considered part of the nursing group, but also had specific tasks outside the field of traditional clinical work. Hence, it was hard for them to view themselves as 'valuable contributors to shared projects', which was considered to be of key importance for recognition in the solidarity sphere. ${ }^{[19]}$ Formal differences in assigned tasks and the MSN graduates' dependence on being allowed to work with research and developmental tasks could also generate a feeling of inferiority in the MSN graduates.

Furthermore, our findings showed that in some cases the managers did not know how to use the MSN graduates' skills or were unable to give priority to their work with research and development. These barriers made the MSN graduates' division between clinical tasks and research and development even more complicated. A collective case study showed how nurse managers tend to have difficulties in including research by academic nurses in clinical practice because the managers tend to prioritise urgent daily demands. ${ }^{[21]}$ Spending time on research and development seems to be considered an extravagance in a clinical setting, despite requests for graduate skills over the years. ${ }^{[8]}$ However, a classical ethnography by Hansen ${ }^{[22]}$ shows how clinical nurses tend to spend time on 
paperwork, such as documentation and preparing the ward round, on days where they have plenty of time. This is contrary to what clinical nurses claim regarding busy days, where they express a common desire to be able to spend more time with the patients. ${ }^{[22]}$ This inner logic, where 'theoretical work' is seen as something to be reserved for quiet days, is transferred to the work of the MSN graduates, even though their tasks differ. The relationship between workload and nurses' research utilisation was investigated by Thompson and colleagues ${ }^{[23]}$ who conclude that the nurses' perception of lack of time is constituted in a multi-varied understanding of physical and psychological dimensions. Thompson and colleagues ${ }^{[23]}$ recommend reducing the focus on workload when advancing evidence-based nursing, if research is expected to be recognised as part of clinical nursing practice.

\section{Strengths and imitations}

A key strength of this study was that the participants were very articulate and willing to share their experiences. According to Kvale and Brinkamnn, ${ }^{[24]} 15$ interviewees should be an appropriate number to provide enough variations in the data material to describe a given phenomenon in detail in a qualitative interview study. However, a potential limitation is that all the participants had the same educational background and may therefore have the same views on research and its utilisation. Also, the authors are all employed at the university where the MSN graduates were educated, but did not however have any personal relationships with the students.

\section{Conclusion}

Our study shows how Masters of Science in Nursing struggled to use their academic skills in clinical practice six months after graduating, despite their enthusiasm to do so. Even though the MSN graduates were highly motivated, they felt the need to camouflage their research-related tasks; they had to battle with a lack of time and a lack of support, rather than a lack of knowledge, which was stated as a barrier for non-master's nurses engaging in research. Instead, the MSN graduates were met by barriers of time restrictions in their dual part-time positons in research and patient care with limited time to spend on research tasks, where the culture in the departments had direct patient care as the main interest, and where research activities lacked support, recognition and solidarity by their management and colleagues.

The findings will have an impact in clinical practice by informing newly-graduated MSN nurses about the possibilities and barriers they may meet, and by informing nurse managers about the possibilities of utilising the MSN graduates' academic competencies. In order to create a space for engaging in nursing research-related tasks, academic nurses must adhere to the norms of the nursing culture by staying close to practice. There is a need for evidence-based practice in which the nursing management plays an important role as the vanguard of nursing research to improve patient outcomes in clinical practice.

\section{ACKNOWLEDGEMENTS}

We would like to thank the participating MSN graduates for their time and efforts.

\section{CONFLicts OF INTEREST Disclosure}

The authors declare that there is no conflict of interest.

\section{REFERENCES}

[1] Breimaier HE, Halfens RJ, Lohrmann C. Nurses' wishes, knowledge, attitudes and perceived barriers on implementing research findings into practice among graduate nurses in Austria. J Clin Nurs. 2011; 20(11-12): 1744-1756. PMid:21362075 https://doi.org/10.1 $111 / j .1365-2702.2010 .03491 . x$

[2] Renolen $\AA$, Hjälmhult E. Nurses experiences of using scientific knowledge in clinical practice: a grounded theory study. Scand J Car Scien. 2015; 29: 633-641. PMid:25753736 https ://doi .or $\mathrm{g} / 10.1111 / \mathrm{scs} .12191$

[3] Chan GK, Barnason S, Dakin CL, et al. Barriers and Perceived Needs for Understanding and Using Research Among Emergency Nurses. J Emerg Nurs. 2011; 37(1): 24-31. PMid:21237364 https : //doi.org/10.1016/j.jen.2009.11.016

[4] Kajermo KN, Boström AM, Thompson DS, et al. The BARRIERS scale - the barriers to research utilization scale: A systematic review. Implem Scien. 2010; 5: 32. PMid:20420696 https: //doi.org/10.1186/1748-5908-5-32
[5] Carlson CL, Plonczynski DJ. Has the BARRIERS Scale changed nursing practice? An integrative review. J Adv Nurs. 2008; 63(4): 322-333. PMid:18727758 https://doi.org/10.1111/j.1365 $-2648.2008 .04705 . x$

[6] Renolen Å, Høye S, Hjälmhult E, et al. "Keeping on track" - Hospital nurses' struggles with maintaining workflow while seeking to integrate evidence-based practice into their daily work: A grounded theory study. Int J Nurs Stud. 2018; 77: 179-188. PMid:29100200 https://doi.org/10.1016/j.ijnurstu.2017.09.006

[7] Brown CE, Ecoff L, Kim SC, et al. Multi-institutional study of barriers to research utilisation and evidence-based practice among hospital nurses. J Clin Nurs. 2010; 19(13-14): 1944-1951. PMid:20920021 https://doi.org/10.1111/j.1365-2702.2009.03184.x

[8] Clark L, Casey D, Morris S. The value of Master's degrees for registered nurses. Brit J Nurs. 2015; 24(5): 16-20. PMid:25815824 https://doi.org/10.12968/bjon.2015.24.6.328

[9] Cotterill-Walker SM. Where is the evidence that master's level nursing education makes a difference to patient care? A literature 
review. Nurse Educ Today. 2012; 32(1): 57-64. PMid:21371793

https://doi.org/10.1016/j.nedt.2011.02.001

[10] Ge S, Xi X, Guo G. A systematic review of the impact of master'seducated nurses on inpatient care. Int J Nurs Scien. 2015; 2(4): 414421. https://doi.org/10.1016/j.ijnss.2015.10.003

[11] Aiken LH, Sloane DM, Bruyneel L, et al. Nurse staffing and education and hospital mortality in nine European countries: a retrospective observational study. The Lancet. 2014; 383(9931): 1789-1790. https ://doi.org/10.1016/S0140-6736(13)62631-8

[12] Drennan J. Professional and academic destination of masters in nursing graduates: A national survey. Nurse Educ Today. 2008; 28(6): 751-759. PMid:18242790 https ://doi.org/10.1016/j. nedt. 2007.12 .003

[13] Baird LMG, Miller T. Factors influencing evidence-based practice for community nurses. Brit J Comm Nurs. 2015; 20(5). PMid:25993372 https://doi.org/10.12968/bjcn.2015.20.5.233

[14] Van Oostveen CJ, Goedhart NS, Francke AL, et al. Combining clinical practice and academic work in nursing: A qualitative study about perceived importance, facilitators and barriers regarding clinical academic careers for nurses in university hospitals. J Clin Nurs. 2017; 26(23-24): 4973-4984. PMid:28793367 https : //doi .org/10.1 $111 /$ jocn. 13996

[15] Graneheim UH, Lundman B. Qualitative content analysis in nursing research: concepts, procedures and measures to achieve trustworthiness. Nurse Educ Today. 2004; 24: 105-112. PMid:14769454 https://doi.org/10.1016/j.nedt.2003.10.001
[16] Berthelsen C, Martinsen B, Vamosi M. The positive impact over time of Master's level education on nurses' utilization of nursing researchrelated tasks in clinical practice - A longitudinal cohort study. J Nurs Edu Prac. 2020; 10(4).

[17] Lincoln YS, Guba EG. Naturalistic Inquiry. London: SAGE; 1985.

[18] Hamran T. Pleiekulturen: en utfordring til den teknologiske tenkemåten [Care culture: a challenge for technological thinking]. Oslo: Gyldendal Norsk Forlag; 1991.

[19] Honneth A. Behovet for anerkendelse [The need for recognition]. København: Hans Reitzels Forlag; 1995.

[20] Higgins I, Parker V, Keatinge D, et al. Doing clinical research: The challenges and benefits. Contemp Nurse. 2010; 35(2): 171-181. PMid:20950198 https://doi.org/10.5172/conu.2010.35.2 .171

[21] Hølge-Hazelton B, Kjerholt M, Berthelsen CB, et al. Integrating nurse researchers in clinical practice - a challenging, but necessary task for nurse leaders. J Nurs Manag. 2016; 24(4): 465-474. PMid:26667268 https://doi.org/10.1111/jonm. 12345

[22] Hansen HP. I grænsefladen mellem liv og død [In the interface between life and death]. København: Gyldendal; 1995.

[23] Thompson DS, O'Leary K, Jensen E, et al. The relationship between busyness and research utilization: it is about time. J Clin Nurs. 2008; 17(4): 539-548. PMid:18205684 https://doi.org/10.1111/j . 1365-2702.2007.01981.x

[24] Kvale S, Brinkmann S. InterView. Introduktion til et håndværk [InterViews]. København: Hans Reitzels Forlag; 2015. 